\title{
Joint Sparsity with Partially Known Support and Application to Ultrasound Imaging
}

\author{
Adrien Besson, Student Member, IEEE, Dimitris Perdios, Student Member, IEEE, Yves Wiaux, \\ and Jean-Philippe Thiran, Senior Member, IEEE,
}

\begin{abstract}
We investigate the benefits of known partial support for the recovery of joint-sparse signals and demonstrate that it is advantageous in terms of recovery performance for both rank-blind and rank-aware algorithms. We suggest extensions of several joint-sparse recovery algorithms, e.g. simultaneous normalized iterative hard thresholding, subspace greedy methods and subspace-augmented multiple signal classification (MUSIC) techniques. We describe a direct application of the proposed methods for compressive multiplexing of ultrasound (US) signals. The technique exploits the compressive multiplexer architecture for signal compression and relies on joint-sparsity of US signals in the frequency domain for signal reconstruction. We validate the proposed algorithms on numerical experiments and show their superiority against state-of-the-art approaches in rankdefective cases. We also demonstrate that the techniques lead to a significant increase of the image quality on in vivo carotid images compared to reconstruction without partially known support. The supporting code is available on https://github.com/AdriBesson/ spl2018_joint_sparse.
\end{abstract}

Index Terms-Compressed Sensing, Ultrasound, Greedy Algorithms, Joint Sparsity, MUSIC,

\section{INTRODUCTION}

C OMPRESSED SENSING (CS) [1], [2] aims at solving a single measurement vector (SMV) problem where one would like to retrieve a $k$-sparse vector $\boldsymbol{x} \in \Sigma_{k}$ from measurements $\boldsymbol{y}=\boldsymbol{A} \boldsymbol{x} \in \mathbb{K}^{m}$ where $\boldsymbol{A} \in \mathbb{K}^{m \times n}, \Sigma_{k}=$ $\left\{\boldsymbol{x} \in \mathbb{R}^{n}|| \operatorname{supp}(\boldsymbol{x}) \mid \leq k\right\}$ and $\operatorname{supp}(\boldsymbol{x})$ denotes the support of $\boldsymbol{x}$.

Distributed CS extends CS to the multiple measurement vectors (MMV) problem [3], [4] whose purpose is to recover multiple sparse vectors $\boldsymbol{X}=\left[\boldsymbol{x}_{1}, \boldsymbol{x}_{2}, \ldots, \boldsymbol{x}_{N}\right] \in \mathbb{K}^{n \times N}$ from measurements $\boldsymbol{Y}=\boldsymbol{A} \boldsymbol{X} \in \mathbb{K}^{m \times N}$ [5]. Under the assumption that the signals $\boldsymbol{x}_{i}, i=1, \ldots, N$, share the same support (JSM2 model in [5]), the MMV problem can be written as

$$
\min _{\boldsymbol{X} \in \mathbb{K}^{n \times N}}\|\boldsymbol{X}\|_{0, \text { row }} \text { subject to } \boldsymbol{Y}=\boldsymbol{A} \boldsymbol{X},
$$

where $\|\boldsymbol{X}\|_{0 \text {,row }}$ counts the number of non-zero rows of $\boldsymbol{X}$. Many techniques have been introduced to tackle the MMV

A. Besson, D. Perdios and J.-Ph. Thiran are with the Signal Processing Laboratory 5 (LTS5), Ecole Polytechnique Fédérale de Lausanne, CH-1015, Lausanne, Switzerland. Y. Wiaux is with the Institute of Sensors, Signals and Systems, Heriot-Watt University, EH14 4AS Edinburgh, United-Kingdom. J.$\mathrm{Ph}$. Thiran is also with the Department of Radiology, University Hospital Center (CHUV) and University of Lausanne (UNIL), CH-1011, Lausanne, Switzerland. This work was supported in part by the UltrasoundToGo RTD project (no. 20NA21_145911) funded by Nano-Tera.ch with Swiss Confederation financing. This work was also supported by the Swiss SNF project number 205320_175974. problem. A first group exploits the multiple signal classification (MUSIC) algorithm, popular in array signal processing. Indeed, Feng and Bresler [6] demonstrate that $\boldsymbol{X}$ can be retrieved using a singular value decomposition of $\boldsymbol{Y}$ in the full-rank case. Extensions of the MUSIC algorithm to rank-defective cases have been proposed such as subspace-augmented MUSIC (SA-MUSIC) [7], compressive MUSIC (CS-MUSIC) [8] and semi-supervised MUSIC [9]. The second group of techniques extend algorithms developed for standard CS to the MMV case. Mixed-norm algorithms exploit extensions of $\ell_{1}$-minimization algorithms [10], [11], [12]. Several greedy algorithms have also been extended leading to simultaneous orthogonal matching pursuit [13], [14], simultaneous normalized hard thresholding pursuit [15], [16], simultaneous compressive sampling matching pursuit [16] and simultaneous normalized iterative hard thresholding (SNIHT) [16]. In [17], Davies and Eldar introduce rank-aware orthogonal recursive matching pursuit (RA-ORMP), a greedy method which exploits the rank information of $\boldsymbol{X}$. Lee et al. [7] propose the orthogonal subspace matching pursuit (OSMP), very similar to RA-ORMP.

CS with partially known support consists in injecting a prior knowledge of the support of the unknown signal into the CS problem resulting in fewer necessary measurements than standard CS. The concept has been developed for the SMV problem by Vaswani and $\mathrm{Lu}$ [18] and extended by Jacques [19]. Carrillo et al. [20], [21] have also suggested extensions of various greedy algorithms.

In this work, we propose to study the benefits of known partial support on the performance of joint-sparse recovery algorithms. In Section II, we present uniqueness conditions for the solution of Problem (1) in case of partially known support. We also propose extensions of several algorithms, i.e. SNIHT, RA-ORMP, OSMP and subspace augmented MUSIC methods. In Section III, we show an application of the proposed algorithms to the recovery of ultrasound (US) signals from multiplexed measurements. The proposed methods are validated through numerical experiments and applied on in vivo carotid US images in Section IV. Concluding remarks are given in Section V.

\section{Joint Sparsity With Partially KNOWN Support}

\section{A. Notation}

Symbol $\mathbb{K}$ denotes a scalar field, e.g. $\mathbb{R}$ or $\mathbb{C}$. Given a space $\mathcal{I} \subset \mathbb{K}^{N}, \operatorname{dim}(\mathcal{I})$ designates its dimension, $\mathbf{P}_{I}$ and $\mathbf{P}_{I}^{\perp}$ define the projectors onto $\mathcal{I}$ and its orthogonal complement 
$\mathcal{I}^{\perp}$. Similarly, given a set of integers $J \subset\{1, \ldots, n\}, \bar{J}=$ $\{1, \ldots, n\} \backslash J$ denotes its complement and $|J|$ its cardinality. The Hermitian transpose of a matrix $\boldsymbol{X}$ is denoted by $\boldsymbol{X}^{*}$ and the Moore pseudo-inverse by $\boldsymbol{X}^{\dagger} .\|\boldsymbol{X}\|_{F}$ is the Frobenius norm of $\boldsymbol{X} . \boldsymbol{X}_{\left(J_{0}\right)}$ (resp. $\boldsymbol{X}_{J_{0}}$ ) is the sub-matrix formed by the restriction of $\boldsymbol{X}$ to the rows (resp. the columns) indexed by $J_{0}$. The space spanned by the columns of $\boldsymbol{X}$ is defined by $\mathcal{R}(\boldsymbol{X})$. The rank of $\boldsymbol{X}$ is designated by $\operatorname{rank}(\boldsymbol{X})$ and $\operatorname{spark}(\boldsymbol{X})$ defines its spark i.e. the smallest number of columns from $\boldsymbol{X}$ that are linearly dependent. We use $\operatorname{supp}(\boldsymbol{X})$ as the rowsupport of $\boldsymbol{X}$ and $\Sigma_{k}^{(n, N)}$ as the set of $k$-row-sparse matrices of $\mathbb{K}^{n \times N}$. We also refer the reader to the definition of the upper and lower asymmetric restricted isometry (ARIP) constants of order $k$ [16], denoted as $U_{k}$ and $L_{k}$ and whose definitions are given in supplementary material of this work. In the remainder, we are interested in recovering $\boldsymbol{X} \in \Sigma_{k}^{(n, N)}$ such that $\operatorname{supp}(\boldsymbol{X})=J=J_{0} \cup J_{1}$, with $J_{0} \subset\{1, \ldots, n\}$ and $J_{1} \subset \bar{J}_{0}$, from measurements $\boldsymbol{Y}=\boldsymbol{A} \boldsymbol{X}$, with $\boldsymbol{A} \in \mathbb{K}^{m \times n}$. We assume that $J_{0}$ is known a priori.

\section{B. Uniqueness of the $\ell_{0}$-norm Minimization}

In this section, we extend the uniqueness condition derived by Vaswani and Lu [18] to the MMV problem. The objective is to establish guarantees of uniqueness of the solutions in the case of MMV problems with partially known support that are weaker than the ones for standard MMV problems [3], [4]. In order to do that, we reformulate the problem with partially known support as:

$$
\min _{\boldsymbol{X} \in \mathbb{K}^{n \times N}}\left\|\boldsymbol{X}_{\left(\bar{J}_{0}\right)}\right\|_{0 \text {,row }} \text { subject to } \boldsymbol{Y}=\boldsymbol{A} \boldsymbol{X} .
$$

The following theorem gives an upper bound on the sparsity for the MMV problem with partially known support in the general case.

Theorem 1. The matrix $\boldsymbol{X}$, with $\operatorname{supp}(\boldsymbol{X})=J_{0} \cup J_{1}, J_{0}$ known, will be the unique solution of (2), if $\boldsymbol{Y}=\boldsymbol{A} \boldsymbol{X}$ and

$$
k<\frac{\operatorname{spark}(\boldsymbol{A})+\left|J_{0}\right|}{2} \text {. }
$$

Proof. Define $\boldsymbol{X}^{1} \in \mathbb{K}^{n \times N}$ and $\boldsymbol{X}^{2} \in \mathbb{K}^{n \times N}$ such that $\boldsymbol{X}^{1} \neq$ $X^{2}$ and both are solutions of (2). Consider that the rows of $\boldsymbol{X}^{1}\left(\right.$ resp. $\left.\boldsymbol{X}^{2}\right)$ are supported on $J_{0} \cup \Delta_{1}$ (resp. $J_{0} \cup \Delta_{2}$ ) such that $\left|\Delta_{1}\right|=\left|\Delta_{2}\right|=u$. Thus, the rows of $\boldsymbol{X}^{1}-\boldsymbol{X}^{2}$ are supported on $J_{0} \cup \Delta_{1} \cup \Delta_{2}$ and we can write the following equivalence

$$
\boldsymbol{A}_{J_{0} \cup \Delta_{1} \cup \Delta_{2}}\left(\boldsymbol{X}^{1}-\boldsymbol{X}^{2}\right)_{\left(J_{0} \cup \Delta_{1} \cup \Delta_{2}\right)}=0 \Leftrightarrow \boldsymbol{X}^{1}=\boldsymbol{X}^{2},
$$

if $\operatorname{spark}(\boldsymbol{A})>\left|J_{0}\right|+2 u \geq\left|J_{0} \cup \Delta_{1} \cup \Delta_{2}\right|$. Using the fact that $k=\left|J_{0}\right|+u$, (3) holds.

Theorem 1 is an extension to the MMV problem of Proposition 1 of [18] and the upper bound is the same as for the SMV problem. At this point, it would be beneficial to combine the information on $\operatorname{rank}(\boldsymbol{Y})$ and the partially known support to relax the uniqueness condition provided for rank-aware algorithms [3], [6], [17]. To do so, we remind the following lemma.
Lemma 1 (Theorem 2.4 of [3]). The matrix $X$ will be the unique solution of (1), if $\boldsymbol{Y}=\boldsymbol{A} \boldsymbol{X}$ and

$$
k<\frac{\operatorname{spark}(\boldsymbol{A})+\operatorname{rank}(\boldsymbol{Y})-1}{2} .
$$

We can now state the main claim of the section:

Theorem 2. The matrix $\boldsymbol{X}$, with $\operatorname{supp}(\boldsymbol{X})=J_{0} \cup J_{1}, J_{0}$ known, will be the unique solution of (1), if $\boldsymbol{Y}=\boldsymbol{A} \boldsymbol{X}$ and

$$
k<\frac{\operatorname{spark}(\boldsymbol{A})+\operatorname{rank}\left(\boldsymbol{Y}_{a}\right)-1}{2},
$$

where $\boldsymbol{Y}_{a}=\left[\boldsymbol{Y}, A_{J_{0}}\right]$.

Proof. Consider $\boldsymbol{Y}_{a}=\left[\boldsymbol{Y}, \boldsymbol{A}_{J_{0}}\right]$. We define the augmented signal matrix $\boldsymbol{X}_{a}=\left[\boldsymbol{X}, \boldsymbol{I}_{J_{0}}\right]$, where $\boldsymbol{I}_{J_{0}} \in \mathbb{R}^{n \times\left|J_{0}\right|}$ is the identity with columns restricted to $J_{0}$, such that $\boldsymbol{Y}_{a}=\boldsymbol{A} \boldsymbol{X}_{a}$. We can see that $\operatorname{supp}\left(\boldsymbol{X}_{a}\right)=\operatorname{supp}(\boldsymbol{X})$. Thus, by applying Lemma 1 and if (6) holds, $\boldsymbol{X}_{a}$ is the unique solution of the augmented MMV problem and $\boldsymbol{X}$ is therefore the unique solution of (1).

Theorem 2 can be interpreted in terms of subspace augmentation discussed in Proposition 5.6 of [7]. Indeed, the partially known support $J_{0}$ is used to augment the signal subspace $\mathcal{R}(\boldsymbol{Y})$ with basis vectors of $\mathcal{R}\left(\boldsymbol{A}_{J_{0}}\right)$. Thus, it is advantageous when some of the basis vectors of $\mathcal{R}\left(\boldsymbol{A}_{J_{0}}\right)$ are orthogonal to $\mathcal{R}(\boldsymbol{Y})$. Now that we have established uniqueness conditions, we propose extensions of existing joint sparse algorithms to partially known support.

\section{Greedy Methods With Partially Known Support}

1) RA-ORMP and OSMP: RA-ORMP and OSMP are very similar as explained in [7] and the argument detailed below may be applied to both algorithms. For conciseness, we focus on RA-ORMP in the remainder. The partially known support can be exploited in the initialization step of the RA-ORMP algorithm [17]. The idea is to consider $J_{0}$ as the initial support in the algorithm and perform the following initialization:

$$
\begin{aligned}
\boldsymbol{R}^{0} & =\mathbf{P}_{\mathcal{R}\left(\boldsymbol{A}_{J_{0}}\right)}^{\perp} \boldsymbol{Y} \\
\boldsymbol{\Phi} & =\mathbf{P}_{\mathcal{R}\left(\boldsymbol{A}_{J_{0}}\right)}^{\perp} \boldsymbol{A}, \tilde{\boldsymbol{\Phi}}_{n}=\boldsymbol{\Phi}_{n} /\left\|\boldsymbol{\Phi}_{n}\right\|_{2}, \forall n \notin J_{0},
\end{aligned}
$$

where the notations $\boldsymbol{\Phi}$ and $\tilde{\boldsymbol{\Phi}}_{n}$ are used to be consistent with [17]. The RA-ORMP procedure initalized with the above steps is denoted as RA-ORMP-PKS. The remaining steps of RA-RORMP-PKS are the same as RA-ORMP (Algorithm 3 of [17]) and aim at recovering the unknown support $J_{1}$. Regarding the recovery, RA-ORMP-PKS is guaranteed to recover $\boldsymbol{X}$ from $\boldsymbol{Y}$ in the noiseless case provided that $\operatorname{rank}\left(\boldsymbol{Y}_{a}\right)=k$ (by application of Theorem 6 of [17] to the augmented matrix $\boldsymbol{Y}_{a}$ ).

2) SNIHT: SNIHT proposes an extension of the iterative hard thresholding (IHT) algorithm [22] to the MMV problem [16]. Based on the extension of IHT to partially known support [21], we suggest SNIHT-PKS in which steps $7-9$ of SNIHT (Algorithm 1 in [16]) are replaced by the following:

$$
\boldsymbol{X}^{i}=\mathcal{H}_{k-\left|J_{0}\right|}^{J_{0}}\left(\boldsymbol{X}^{i-1}+\omega\left(\boldsymbol{A}^{*} \boldsymbol{R}^{i-1}\right)\right),
$$

where $\omega \in \mathbb{R}$ and the non-linear operator $\mathcal{H}_{k}^{J}$ (.) is defined for $J \subset\{1, \ldots, n\}$ as

$$
\mathcal{H}_{k}^{J}(\boldsymbol{X})=\boldsymbol{X}_{(J)}+\mathcal{H}_{k}\left(\boldsymbol{X}_{(\bar{J})}\right)
$$


where $\mathcal{H}_{k}(\boldsymbol{X})$ is the hard-thresholding operator which selects the $k$ rows of $\boldsymbol{X}$ with largest row- $\ell_{2}$-norm. We state the main result of this section, which provides an upper bound on the discrepancy between the output of SNIHT-PKS and the optimal row-sparse approximation of the solution.

Theorem 3 (Simultaneous Sparse Approximation with Partially Known Support). Consider that $\boldsymbol{Y}=\boldsymbol{A} \boldsymbol{X}_{\left(J_{0} \cup J_{1}\right)}+\tilde{\boldsymbol{E}}$. If $\boldsymbol{A}$ satisfies the following ARIP conditions: $2 U_{c k}+2 L_{c k}+L_{k}<1$ where $c \in \mathbb{N}$ is such that $c k \geq 3 k-2\left|J_{0}\right|$, then the error of SNIHT-PKS at iteration $i$ is bounded by:

$$
\left\|\boldsymbol{X}^{i}-\boldsymbol{X}_{(J)}\right\|_{F} \leq \alpha^{i}\left\|\boldsymbol{X}_{(J)}\right\|_{F}+\frac{\beta}{1-\alpha}\|\tilde{\boldsymbol{E}}\|_{F},
$$

where $\alpha=2 \frac{U_{c k}+L_{c k}}{1-L_{k}}, \beta=2 \frac{\sqrt{1-U_{d k}}}{1-L_{k}}$ and $d \in \mathbb{N}$ is such that $d k \geq 2 k-\left|J_{0}\right|$.

The proof is given in the supplementary material of the proposed work. It can be seen that the results are closed to the one obtained by Carillo et al. [21] for the SMV case, in which the matrix $\boldsymbol{A}$ has to meet the RIP property of order $3 k-$ $2\left|J_{0}\right|$. In addition, the ARIP conditions provided by Theorem 3 are weaker than the ones of SNIHT, which can be translated into fewer measurements necessary to fulfill (11). Howver, compared to the bound established in Theorem 1, SNIHT-PKS requires $\boldsymbol{A}$ to be $c k$-RIP which is stronger than spark $(\boldsymbol{A})>$ $2 k-\left|J_{0}\right|$.

\section{MUSIC-based Methods with Partially Known Support}

MUSIC-based algorithms exploit additional information provided by the signal subspace to help the recovery of $\boldsymbol{X}$ [6]. In the case of partially known support, we rely on the augmented measurement matrix $\boldsymbol{Y}_{a}$ rather than $\boldsymbol{Y}$ and we use the following criterion to identify $\operatorname{supp}(\boldsymbol{X}): \forall j \in \bar{J}_{0}$, $j \in J_{1}$ if and only if $\boldsymbol{Q}_{a}^{*} \boldsymbol{A}_{j}=0$ and $\operatorname{rank}\left(\boldsymbol{Y}_{a}\right)=k$, where $\boldsymbol{Q}_{a} \in \mathbb{R}^{m \times(m-k)}$ is an orthonormal basis of $\mathcal{R}\left(\boldsymbol{Y}_{a}\right)^{\perp}$.

In the rank-defective case where $\operatorname{rank}\left(\boldsymbol{Y}_{a}\right)<k$, we first identify $k-\operatorname{rank}(\boldsymbol{Y})$ components of $\operatorname{supp}(\boldsymbol{X})$ using a greedy algorithm initialized with the partially known support. The remaining $\operatorname{rank}(\boldsymbol{Y})$ components either come from the signal subspace (SA-MUSIC [7]) or are identified based on a generalized MUSIC criterion (CS-MUSIC [8]). We use the partially known support to initialize the greedy algorithm since the success of subspace augmented methods relies on the successful partial support recovery of the greedy algorithm and it is known that forward selection approaches perform far better when smaller subsets have to be recovered [7].

\section{Multiplexing OF Ultrasound Signals}

High-quality 3D US imaging necessitates a US probe of more than thousands of transducer elements and the ability to perform electronic focusing would require as many coaxial cables connecting the probe to the back-end system resulting in very cumbersome equipments. In order to reduce the amount of cables, sparse array techniques where a subset of transducer elements are used for imaging have been widely studied [23]. Many different strategies have been designed such as random sparse arrays [24], [25], Vernier arrays [26], row-column addressed arrays [27], [28], [29] and free-hand [30] or motorized [31] 1D arrays. Alternatively, pre-beamforming methods, where part of the imaging process is achieved in the head of the probe [32], and time multiplexing techniques have been investigated for both dense and sparse arrays [32], [33], [34]. While proposing a drastic reduction on the number of transducer elements, such methods have a significant impact either on the image quality [23] or on the frame rate.

In this section, we describe a direct application of the proposed algorithms for compressive multiplexing of US signals. More precisely, we propose to exploit the compressive multiplexer (CMUX) [35] architecture to reduce both the number of analog cables connecting the US probe to the back-end system and the number of analog-to-digital converters (ADC). We consider a US probe made of $N$ transducer elements which receive signals as backscattered echoes from a previously insonified medium, at a rate $\Omega$ during a time $T$. The set of those signals is denoted as element raw-data and stored as $\boldsymbol{M} \in \mathbb{R}^{n \times N}$, where $n=T \Omega$. In the proposed architecture, described in Figure 1, we equip the head of the probe with $N_{c}$ CMUX, each of which working at $\Omega$ and compressing $N_{t}$ signals, with $N=N_{t} N_{c}$. Thus, one may require only $N_{c} \ll N$ coaxial cables connecting the probe to the back-end system and only $N_{c} \ll N$ ADC. Formally, the measurements have the following form: $\boldsymbol{Y}=\mathcal{A}(\boldsymbol{M})+\boldsymbol{E}$, where $\mathcal{A}: \mathbb{R}^{n \times N_{t}} \rightarrow \mathbb{R}^{n \times N_{c}}$ is the linear operator associated with the CMUX architecture [35] and $\boldsymbol{E} \in \mathbb{C}^{n \times N_{c}}$ is the noise.

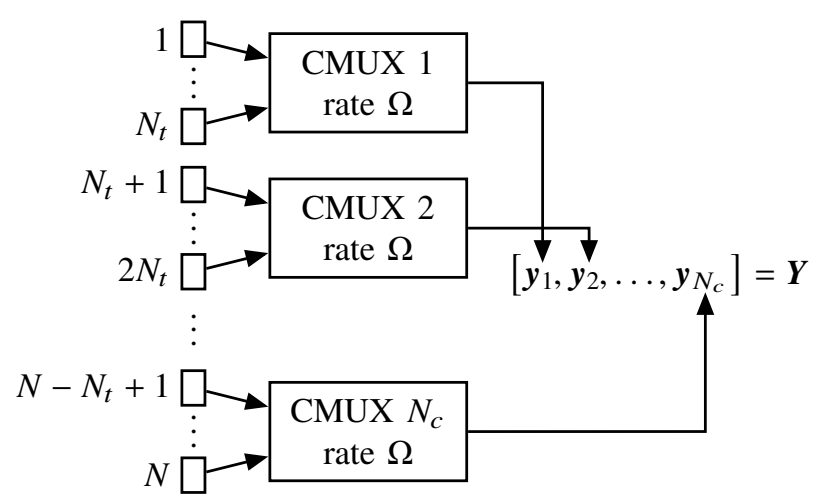

Transducer elements

Fig. 1. Ultrasound Compressive Multiplexer Architecture.

US signals are known to have a relatively sparse spectrum [36], [37] due to the piezo-electric properties of transducer elements and the high sampling frequency required for delay resolution of delay-and-sum beamforming [38]. In addition, we usually have a partial knowledge of such a spectrum which is measured by probe manufacturers. Thus, we are in a scenario where joint sparse algorithms with partially known support can be leveraged. Puts formally, we introduce the 1D discrete Fourier transform $\boldsymbol{F} \in \mathbb{C}^{n \times n}$ and the associated Fourier coefficients $\hat{\boldsymbol{M}}=\boldsymbol{F} \boldsymbol{M}$ such that $\operatorname{supp}(\hat{\boldsymbol{M}})=J_{0} \cup J_{1}$, where $J_{0}$ is the known part of the spectrum, $|\operatorname{supp}(\hat{\boldsymbol{M}})| \ll n$, and we solve the following joint-sparse regularization problem:

$$
\min _{\hat{\boldsymbol{M}} \in \mathbb{C}^{n \times N}}\|\hat{\boldsymbol{M}}\|_{0, \text { row }} \text { subject to } \boldsymbol{Y}=\mathcal{A}\left(\boldsymbol{F}^{*} \hat{\boldsymbol{M}}\right)+\boldsymbol{E} .
$$




\section{EXPERIMENTS}

\section{A. Numerical Experiments}

We explore the empirical performance of SA-MUSIC-PKS, RA-ORMP-PKS and SNIHT-PKS in a noiseless situation and under additive Gaussian noise with a signal-to-noise ratio of $30 \mathrm{~dB}$. We consider a Gaussian random measurement matrix $\boldsymbol{A} \in \mathbb{R}^{m \times n}$, with $\boldsymbol{A}_{i, j} \sim \mathcal{N}(0,1)$, such that $\left\|\boldsymbol{A}_{i}\right\|_{2}=1$ and $n$ is fixed to 128 . The signal matrix $\boldsymbol{X} \in \mathbb{R}^{n \times N}$ is built as a random matrix, with $N=k=30$. 1000 random trials of the algorithms are run for each experiment and the recovery probability is computed as the rate of successful support recovery.

The impact of the partially known support is first analyzed by comparing the recovery probability of SA-MUSIC-PKS for a fixed rank $(s=10)$, for a number of measurements ranging between 30 and 90 , when $0 \%, 25 \%, 50 \%$ and $75 \%$ of the support is known a priori. Then, we compare the methods with their counterpart without known support on two experiments: fixed rank $(s=10)$ for a number of measurements ranging between 30 and 90 and fixed number of measurements $(m=$ 51) for a rank varying between 1 and 30. For both experiments, $75 \%$ of the support is assumed to be known.

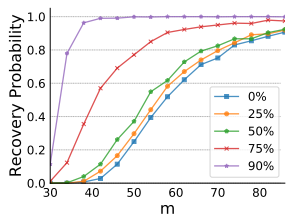

(a)

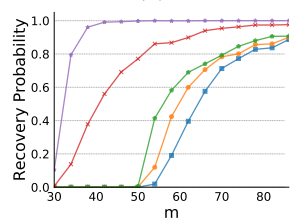

(d)

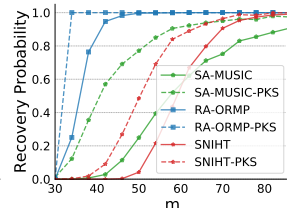

(b)

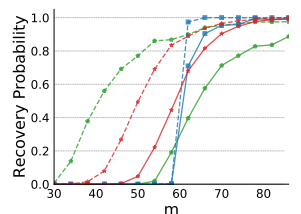

(e)

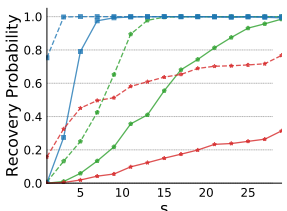

(c)

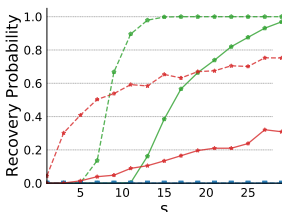

(f)
Fig. 2. (a) and (d) Recovery probability of SA-MUSIC-PKS for varying number of measurements and size of the known support. (b)-(c)-(e)-(f) Recovery probability of SA-MUSIC-PKS, RA-ORMP-PKS and SNIHT-PKS against SA-MUSIC, RA-ORMP and SNIHT for varying number of measurements ((b) and (e)) and varying ranks ((c) and (f)) in a noiseless ((b) and (c)) and in a noisy scenario ((e) and (f)).

On Figs $2 \mathrm{a}$ and $2 \mathrm{~d}$, we can see that SA-MUSIC-PKS is more accurate when larger part of the support is known for both the noiseless and the noisy cases, as expected. On Fig. 2b, we observe that the methods with partially known support achieve significantly better reconstruction than their counterpart without known support in a noiseless scenario which validates the main results of Section II. On Fig. 2e, we see that the conclusions drawn for the noiseless scenario extend to the noisy scenario for SA-MUSIC and SNIHT. Regarding RA-ORMP, we observe that the results in the noisy scenario are significantly lower than in the noiseless scenario due to the fact that the additive noise has a drastic impact on the correlation step involved in RA-ORMP (widely studied in the SMV problem [39]). In this case, RA-ORMP-PKS is only slightly better than RA-ORMP since the algorithm fails to recover the unknown part of the support. Figs. $2 \mathrm{c}$ and $2 \mathrm{f}$ show the benefits of partially known support in terms of the minimal value of $s$ for perfect support recovery. As for Fig. 2b and 2e, we notice that the partially known support significantly helps the recovery of the different algorithms except for RA-ORMP in the noisy case.

Further experiments dedicated to the empirical validation of Theorem 2 are described in the supplementary material.

\section{B. In vivo Ultrasound Signals}

US signals from in vivo carotids have been acquired using a Verasonics US scanner (Redmond, WA, USA) equipped with the ATL-L7-4 probe, operating at $5.2 \mathrm{MHz}$ with $60 \%$ bandwidth and 128 transducer elements. The CMUX architecture is simulated off-line using Python and works at $62.5 \mathrm{MHz}$, with a multiplexing ratio $1 / 8$. On the reconstruction side, we use SNIHT-PKS with 500 iterations. The reasons for the choice of SNIHT-PKS are three-fold. First, the high rankdeficiency $(N \ll n)$ motivates the use of rank-blind algorithms. In addition, SNIHT-PKS has been theoretically proved to be robust to noise (Theorem 3). Finally, the data are complexvalued and relatively high dimensional which prevents the use of pseudo-inverse or EVD, necessary for RA-ORMP and SAMUSIC. We assume that $J_{0}$ contains the indices of the frequencies lying between $2.9 \mathrm{MHz}$ and $6 \mathrm{MHz}$ which corresponds to $85 \%$ of the signal energy. Delay-and-sum beamforming is applied on the recovered US signals followed by envelope detection, normalization and log-compression. The reference log-compressed B-mode image is displayed in Fig. 3a. The images corresponding to the reconstructions with SNIHT-PKS and SNIHT are given in Figs. $3 b$ and 3c.

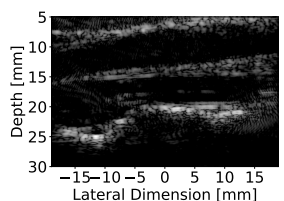

(a) Reference

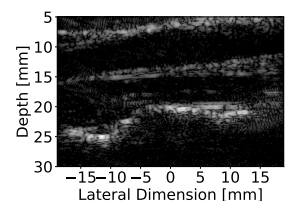

(b) SNIHT-PKS

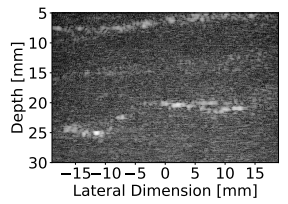

(c) SNIHT
Fig. 3. Log-compressed B-mode images $(40 \mathrm{~dB})$ of an in-vivo carotid. (a)-Reference. (b)-Reconstructed with SNIHT-PKS (PSNR = $45 \mathrm{~dB})$. (c)Reconstructed with SNIHT $($ PSNR $=28 \mathrm{~dB})$.

Both visual assessment and values of the peak-signal-tonoise-ratio (PSNR), computed on the B-mode image and reported in the caption of Fig. 3, show the superior quality of the reconstruction with SNIHT-PKS compared to SNIHT.

\section{CONCLUSION}

In this work, we investigate the recovery of jointly sparse vectors when partial support is known. We quantify the benefits of the known support in terms of a higher upper bound on the row-sparsity than standard MMV problems. We also suggest adaptations of greedy algorithms as well as MUSICbased methods to incorporate the additional information. We apply the proposed algorithms to the recovery of ultrasound signals from compressed measurements, where the objective is to multiplex signals in order to reduce the number of coaxial cables and ADCs. By exploiting the prior knowledge on the frequency support of the signals, we demonstrate that the proposed algorithms significantly outperform the standard MMV ones. 


\section{REFERENCES}

[1] D. L. Donoho, "Compressed sensing," IEEE Trans. Inf. Theory, vol. 52, no. 4, pp. 1289-1306, 2006.

[2] E. Candes, J. Romberg, and T. Tao, "Robust uncertainty principles: exact signal reconstruction from highly incomplete frequency information," IEEE Trans. Inf. Theory, vol. 52, no. 2, pp. 489-509, 2006.

[3] J. Chen and X. Huo, "Theoretical results on sparse representations of multiple measurement vectors," IEEE Trans. Signal Process., vol. 54, no. 12 , pp. 4634-4643, 2006

[4] S. Cotter, B. D. Rao, and K. Kreutz-Delgado, "Sparse solutions to linear inverse problems with multiple measurement vectors," IEEE Trans. Signal Process., vol. 53, no. 7, pp. 2477-2488, 2005.

[5] M. Duarte, S. Sarvotham, D. Baron, M. Wakin, and R. Baraniuk, "Distributed compressed sensing of jointly sparse signals," in ThirtyNinth Asilomar Conf. on Signals, Syst. Comput., 2005, pp. 1537-1541.

[6] Ping Feng and Y. Bresler, "Spectrum-blind minimum-rate sampling and reconstruction of multiband signals," in 1996 IEEE Int. Conf. Acoust. Speech, Signal Process., vol. 3, 1996, pp. 1688-1691.

[7] K. Lee, Y. Bresler, and M. Junge, "Subspace methods for joint sparse recovery," IEEE Trans. Inf. Theory, vol. 58, no. 6, pp. 3613-3641, 2012.

[8] J. M. Kim, O. K. Lee, and J. C. Ye, "Compressive MUSIC: Revisiting the link between compressive sensing and array signal processing," IEEE Trans. Inf. Theory, vol. 58, no. 1, pp. 278-301, 2012.

[9] Z. Wen, B. Hou, and L. Jiao, "Joint sparse recovery with semisupervised MUSIC," IEEE Signal Process. Lett., vol. 24, no. 5, pp. 629-633, 2017.

[10] J. A. Tropp, "Algorithms for simultaneous sparse approximation. Part II: Convex relaxation," Signal Processing, vol. 86, no. 3, pp. 589-602, 2006.

[11] M. Hyder and K. Mahata, "A robust algorithm for joint-sparse recovery," IEEE Signal Process. Lett., vol. 16, no. 12, pp. 1091-1094, 2009.

[12] Y. C. Eldar and M. Mishali, "Robust recovery of signals from a structured union of subspaces," IEEE Trans. Inf. Theory, vol. 55, no. 11 pp. 5302-5316, 2009

[13] J. A. Tropp, A. C. Gilbert, and M. J. Strauss, "Algorithms for simultaneous sparse approximation. Part I: Greedy pursuit," Signal Processing, vol. 86, no. 3, pp. 572-588, 2006.

[14] J.-F. Determe, J. Louveaux, L. Jacques, and F. Horlin, "On the exact recovery condition of simultaneous orthogonal matching pursuit," IEEE Signal Process. Lett., vol. 23, no. 1, pp. 164-168, 2016.

[15] S. Foucart, "Recovering jointly sparse vectors via hard thresholding pursuit," in Proc. SAMPTA, 2011.

[16] J. D. Blanchard, M. Cermak, D. Hanle, and Y. Jing, "Greedy algorithms for joint sparse recovery," IEEE Trans. Signal Process., vol. 62, no. 7, pp. 1694-1704, 2014.

[17] M. E. Davies and Y. C. Eldar, "Rank awareness in joint sparse recovery," IEEE Trans. Inf. Theory, vol. 58, no. 2, pp. 1135-1146, 2012.

[18] N. Vaswani and W. Lu, "Modified-CS: Modifying compressive sensing for problems with partially known support," IEEE Trans. Signal Process., vol. 58, no. 9, pp. 4595-4607, 2010.

[19] L. Jacques, "A short note on compressed sensing with partially known signal support," Signal Processing, vol. 90, no. 12, pp. 3308-3312, 2010.

[20] R. E. Carrillo, L. F. Polania, and K. E. Barner, "Iterative algorithms for compressed sensing with partially known support," in 2010 IEEE Int. Conf. Acoust. Speech Signal Process., 2010, pp. 3654-3657.

[21] _ - "Iterative hard thresholding for compressed sensing with partially known support," in 2011 IEEE Int. Conf. Acoust. Speech Signal Process., 2011, pp. 4028-4031.

[22] T. Blumensath and M. E. Davies, "Iterative hard thresholding for compressed sensing," Appl. Comput. Harmon. Anal., vol. 27, no. 3, pp. 265-274, 2009.

[23] J. Yen, J. Steinberg, and S. Smith, "Sparse 2-D array design for real time rectilinear volumetric imaging," IEEE Trans. Ultrason. Ferroelectr. Freq. Control, vol. 47, no. 1, pp. 93-110, 2000.

[24] D. Turnbull and F. Foster, "Beam steering with pulsed two-dimensional transducer arrays," IEEE Trans. Ultrason. Ferroelectr. Freq. Control, vol. 38, no. 4, pp. 320-333, 1991.

[25] R. E. Davidsen, J. A. Jensen, and S. W. Smith, "Two-dimensional random arrays for real time volumetric imaging," Ultrason. Imaging, vol. 16, no. 3, pp. 143-163, 1994.

[26] G. Lookwood and F. Foster, "Optimizing the radiation pattern of sparse periodic two-dimensional arrays," IEEE Trans. Ultrason. Ferroelectr. Freq. Control, vol. 43, no. 1, pp. 15-19, 1996.

[27] C. Morton and G. Lockwood, "Theoretical assessment of a crossed electrode 2-D array for 3-D imaging," in IEEE Symp. Ultrason. 2003, 2003, pp. 968-971.
[28] C. H. Seo and J. T. Yen, "A $256 \times 2562$-D array transducer with rowcolumn addressing for 3-D rectilinear imaging," IEEE Trans. Ultrason. Ferroelectr. Freq. Control, vol. 56, no. 4, pp. 837-847, 2009.

[29] H. Bouzari, M. Engholm, C. Beers, M. B. Stuart, S. I. Nikolov, E. V. Thomsen, and J. A. Jensen, "Curvilinear 3-D imaging using row-columnaddressed 2-D arrays with a diverging lens: Feasibility study," IEEE Trans. Ultrason. Ferroelectr. Freq. Control, vol. 64, no. 6, pp. 978-988, 2017.

[30] S. W. Hughes, T. J. D’Arcy, D. J. Maxwell, W. Chiu, A. Milner, J. E. Saunders, and R. J. Sheppard, "Volume estimation from multiplanar 2D ultrasound images using a remote electromagnetic position and orientation sensor," Ultrasound Med. Biol., vol. 22, no. 5, pp. 561-572, 1996.

[31] S. Tong, D. Downey, H. Cardinal, and A. Fenster, "A three-dimensional ultrasound prostate imaging system," Ultrasound Med. Biol., vol. 22, no. 6, pp. 735-746, 1996.

[32] B. Savord and R. Solomon, "Fully sampled matrix transducer for real time 3D ultrasonic imaging," in IEEE Symp. Ultrason., 2003, pp. 945953.

[33] J. Yen and S. Smith, "Real-time rectilinear volumetric imaging," IEEE Trans. Ultrason. Ferroelectr. Freq. Control, vol. 49, no. 1, pp. 114-124, 2002.

[34] J. T. Yen and S. W. Smith, "Real-time rectilinear 3-D ultrasound using receive mode multiplexing," IEEE Trans. Ultrason. Ferroelectr. Freq. Control, vol. 51, no. 2, pp. 216-226, 2004.

[35] J. P. Slavinsky, J. N. Laska, M. A. Davenport, and R. G. Baraniuk, "The compressive multiplexer for multi-channel compressive sensing," in 2011 IEEE Int. Conf. Acoust. Speech Signal Process., 2011, pp. 3980 3983.

[36] C. Quinsac, A. Basarab, and D. Kouame, "Frequency domain compressive sampling for ultrasound imaging," Adv. Acoust. Vib., vol. 2012, pp. $1-16,2012$.

[37] A. Basarab, H. Liebgott, O. Bernard, D. Friboulet, and D. Kouame, "Medical ultrasound image reconstruction using distributed compressive sampling," in 2013 IEEE 10th Int. Symp. Biomed. Imaging, 2013, pp. 628-631.

[38] T. L. Szabo, Diagnostic Ultrasound Imaging: Inside Out, second edition. Elsevier, 2014.

[39] T. T. Cai and L. Wang, "Orthogonal matching pursuit for sparse signal recovery with noise," IEEE Trans. Inf. Theory, vol. 57, no. 7, pp. 46804688,2011 